\title{
Switch to Biological Agent in Psoriasis Significantly Improved Clinical and Patient-Reported Outcomes in Real-World Practice
}

\author{
Jenny M. Norlin ${ }^{\text {a, b }}$ Katarina Steen Carlsson ${ }^{b, c}$ Ulf Persson $^{\text {b, } d}$ \\ Marcus Schmitt-Egenolf ${ }^{a}$ \\ ${ }^{a}$ Division of Dermatology and Venereology, Department of Public Health and Clinical Medicine, Umeå University, \\ Umeå; b Swedish Institute for Health Economics, ${ }^{c}$ Clinical Research, Skåne University Hospital, Department of Clinical \\ Sciences, Lund University, and ${ }^{\mathrm{d} I n s t i t u t e}$ for Economic Research, School of Economics, Lund University, Lund, Sweden
}

\section{Key Words}

Biological agents $\cdot$ Psoriasis $\cdot$ EQ-5D $\cdot$ DLQI $\cdot$ PASI $\cdot$ Register

\begin{abstract}
Background: Although clinical studies have shown efficacy of biological agents in moderate to severe psoriasis, observational studies of real-world effectiveness are rare. Objective: To analyse the psoriasis area and severity index (PASI) and quality of life by the EQ-5D questionnaire and dermatology quality of life index (DLQI) in psoriasis patients who switched from conventional systemic treatment to biological agents in clinical practice. Furthermore, to analyse patient groups with the highest benefit of biological agents. Methods: Longitudinal, observational study based on the Swedish National Registry for Systemic Treatment of Psoriasis, PsoReg. Outcomes of biological-naïve patients who switched to a biological agent $(n=267)$ were analysed before switch and at the first follow-up. Results: Patients significantly improved in EQ-5D, DLQI and PASI ( $p<0.001)$. Patients with DLQI $\geq 10$ and/or PASI $\geq 10$ had the greatest benefits from biological agents in terms of EQ-5D. Conclusions: Patients with moderate to severe psoriasis benefit from biological agents in clinical practice; the patients with the highest benefits were those with high pretreatment PASI and DLQI scores.

Copyright $\odot 2013$ S. Karger AG, Basel
\end{abstract}

\section{KARGER}

E-Mail karger@karger.com

www.karger.com/drm

\section{Introduction}

Since biological agents were introduced for patients with moderate to severe psoriasis, randomized controlled trials (RCTs) have shown substantial benefits of these treatments; both in terms of clinical skin involvement [1, $2]$ and in terms of health-related quality of life (HRQOL) [3-5]. RCTs are the gold standard for testing efficacy and safety in evidence-based medicine. Observational studies, on the other hand, are important when estimating effectiveness, i.e. how interventions work in real-world settings. RCTs may be very different from clinical practice because of study selection criteria. RCTs typically exclude older age groups and patients with comorbidities, and they are limited by short study periods and small samples [6, 7]. Observational studies are necessary in health economic evaluations, in order to ensure that society's money is well spent, but it may also provide a 'reality check' for physicians when individualizing patients' treatments. Observational studies of biological agents for psoriasis are rare [8].

Swedish treatment guidelines state that the indication for biological agents in psoriasis is moderate to severe psoriasis in adults who do not respond to conventional systemic treatment or who show intolerance or contraindications to such treatments $[9,10]$. The National Registry for Systemic Treatment in Sweden, PsoReg, makes it possible to analyse nationwide real-world data, where pa- 
tient characteristics and treatment compliance may be different from RCTs.

There are a number of measures to assess psoriasis [11, $12]$. Whereas the clinical measure psoriasis area and severity index (PASI) and the dermatology-specific HRQOL measure dermatology quality of life index (DLQI) are widely used and valid measures in psoriasis [11-17]; the generic preference-based EQ-5D questionnaire is a good complement [18], essential in cost-effectiveness analyses [19].

The objective of this study was to analyse the three key outcomes EQ-5D, DLQI and PASI in patients with moderate to severe psoriasis who switch from conventional systemic treatment to a biological agent in clinical practice, and to analyse which subgroups of patients benefit most from biological agents.

\section{Patients and Methods}

\section{Study Patients}

PsoReg, the National Registry for Systemic Treatment of Psoriasis in Sweden, was funded in 2007 [20]. Patients were registered at local, regional and university hospitals as well as private praxis and treatment centres driven by the patient organization PSO. There is no explicit information in PsoReg about reasons for switching to biologicals, or for discontinuing treatments. Data was retrieved in May 2011.

\section{Outcome Measures}

The generic preference-based HRQOL measure EQ-5D is based on 5 dimensions: mobility, self-care, usual activities, pain/ discomfort and anxiety/depression [21]. Respondents report (1) no problems, (2) some or moderate problems or (3) extreme problems in each dimension. This results in a health profile, for example '11222' which indicate no problems in mobility or self-care, but some problems with usual activities, pain/discomfort and anxiety/depression. Each health profile is associated with utility weights derived from a previous study [22]. These utility weights were normative assessments by the general population of the 243 possible combinations of health profiles. EQ-5D values can be used to calculate QALYs (quality-adjusted life years), which are necessary in health economic analysis. In this analysis, EQ-5D values below zero were truncated to zero.

The dermatology-specific, HRQOL measure DLQI relates to how the skin disease has affected the person's life over the previous week. The DLQI questionnaire includes 10 questions scored on a 0 - to 3-point scale, which results in a summed value ranging from 0 (best health state) to 30 (worst health state). A DLQI score of $0-1$ has been interpreted as 'no effect on QOL', 2-5 as 'small effect', 6-10 as 'moderate effect', 11-20 as 'very large effect' and 2130 as 'extremely large effect' [23].

The PASI includes the severity of the three main signs of psoriasis on the skin (redness, scaliness and thickness) weighted by the coverage of the affected body part (legs, body, arms and head) [24]. The PASI score ranges from 0 up to 72 in theory, where a higher score stands for a severer skin involvement of psoriasis. PASI 75 (a 75\% improvement in PASI) is usually seen as the clinical end point in RCTs and has even been suggested as treatment goal in clinical practice [25].

Approved biological agents for psoriasis in Sweden at the time of analysis were adalimumab, etanercept, infliximab and ustekinumab.

\section{Analysis and Statistical Methods}

Analysis of outcomes in relation to switch was limited to biological-naïve patients that had at least one assessment of PASI, DLQI and/or EQ-5D before and one assessment after switch from conventional systemic treatment to a biological agent. Observations closest in time to switch for each patient were used. The number of patients who had discontinued treatment with biological agents at the follow-up was reported. A Wilcoxon signed rank test was used to determine whether there was a difference in EQ5D, DLQI and PASI before and after switch to a biological agent. The Wilcoxon signed rank test was used as variables were nonnormally distributed and dependent.

The benefit of the biological agent, in terms of change in EQ$5 \mathrm{D}$, was analysed in different subgroups of patients: subgroups of pretreatment PASI and DLQI intervals of $\leq 10$ compared to $\geq 10$, patients with PASI $>10$ and/or DLQI $>10$ compared to patients with PASI $\leq 10$ and DLQI $\leq 10$; patients with percentage change of PASI 75 compared to patients who did not achieve PASI 75; subgroups of different clinical characteristics of psoriasis (nail involvement, erythrodermia, palmoplantar non-pustular, general pustular, palmoplantar pustular and acrodermatitis continua suppurativa), and subgroups of first biological agent. The MannWhitney $U$ test was used to determine whether there was a significant difference in outcomes between different patient groups.

Sex, age [18] and psoriatic arthropathy [26] are known confounders for HRQOL. Therefore, we controlled for these variables in an ordered logit regression analysis with EQ-5D change as the outcome. For the regression analysis, EQ-5D change was divided into 9 intervals of 1 to $>0.75,0.75$ to $>0.5,0.5$ to $>0.25,0.25$ to $>0$, $0,<0$ to -0.25 etc.).

Three sensitivity analyses were performed. Firstly, analysis included each patient's first assessments within the follow-up time of 12-52 weeks (opposed to the first follow-up after switch). Secondly, patients who discontinued biological agents were excluded. Thirdly, patients with uncommon clinical characteristics (erythrodermia, palmoplantar non-pustular, general pustular, palmoplantar pustular and acrodermatitis continua suppurativa) were excluded.

Patients were assumed to start their treatment when drugs were prescribed by the physician. Drug treatment was assumed to be ongoing until the last day of observation (May 20,2011) unless a prior date of removal was registered. Patients who had received efalizumab as their first biological agent were excluded from the analysis as the drug was withdrawn in 2009.

Statistical analysis was performed using Stata statistical software (release 11.1, College Station, Tex., USA).

\section{Ethical Concerns}

This research was done in adherence to the Declaration of Helsinki guidelines and has been approved by the Umeå Ethical Review Board, Sweden. The project was conducted with informed consent from patients. 
Table 1. Characteristics among patients who switched to a biological agent during registration assessments of PASI, DLQI and/ or EQ-5D before and after switch $(n=267)$

\begin{tabular}{lc}
\hline Men & $173(65)$ \\
Women & $94(35)$ \\
Mean age at the time of switch \pm SD, years & $48 \pm 14.5$ \\
Clinical characteristics & \\
$\quad$ Plaque psoriasis & $248(93)$ \\
Psoriatic arthropathy & $94(35)$ \\
Nail involvement & $84(31)$ \\
Erythroderma & $11(4)$ \\
General pustular psoriasis & $6(2)$ \\
Palm pustular psoriasis & $3(1)$ \\
Non-palm pustular psoriasis & $10(4)$ \\
Acrodermatitis continua suppurativa & $2(1)$ \\
First biological agent & \\
Adalimumab & $82(31)$ \\
Etanercept & $152(57)$ \\
Infliximab & $16(6)$ \\
Ustekinumab & $17(6)$ \\
Patients who had discontinued treatment with & \\
biological agent on first follow-up & $38(14)$ \\
Number of assessments ${ }^{1}$ & \\
Mean before switch & 2.5 \\
Mean after switch & 3.5 \\
Min.-max. & $1-32$ \\
Mean time ${ }^{1}$ between assessments and switch to biological agent \\
\pm SD, days & \\
Time before switch & $27.8 \pm 87.3$ \\
Time to first follow-up & $112.1 \pm 136.0$ \\
Patients with assessments before and after switch & \\
PASI & $261(98)$ \\
DLQI & $251(94)$ \\
EQ-5D & $229(86)$ \\
\hline
\end{tabular}

Figures in parentheses indicate percentages.

${ }^{1}$ Assessments of PASI: number of assessments differed somewhat between EQ-5D, DLQI and PASI as there were missing values.

\section{Results}

When data was retrieved 2,923 patients were registered in PsoReg. There were 267 biological-naïve patients who switched to a biological agent during the period of observation and had assessments of PASI, DLQI and/or EQ-5D before switch and at the follow-up (fig. 1, table 1). There was no statistically significant difference in the distribution of sex between patients who switched to a biological agent during registration compared to patients who had previously used biological agents $(\mathrm{p}=0.802)$. Switchers were somewhat younger than patients who had used biological agents previously, with a mean age of 51 years $(S D=13.7 ; \mathrm{p}=0.02)$.

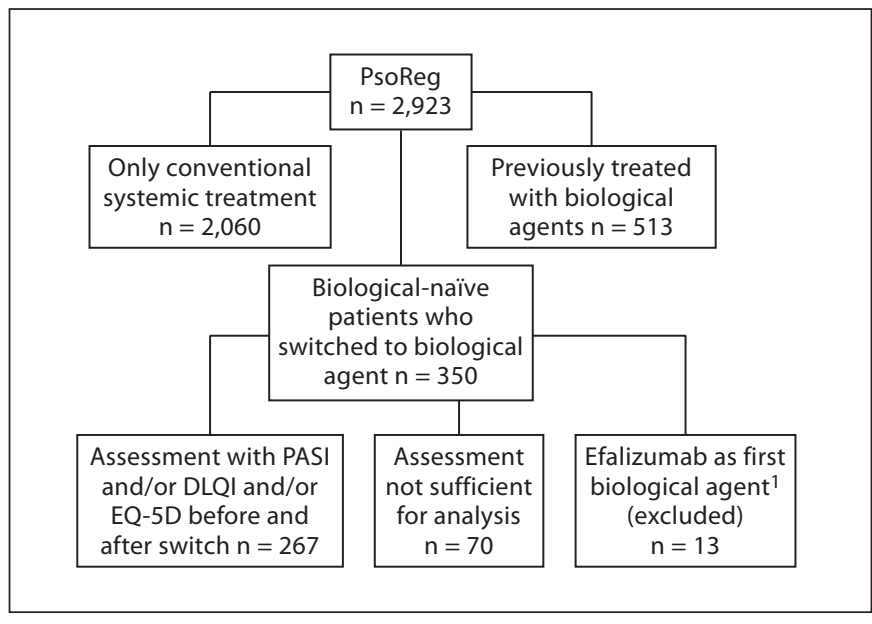

Fig. 1. Number of patients in PsoReg with conventional treatment/biological agents and assessments of PASI, DLQI and EQ5D. ${ }^{1}$ Efalizumab was withdrawn in 2009 and therefore excluded from analyses.

The numbers of PASI, DLQI and EQ-5D assessments differed between patients, as well as the time between assessments (table 1). About $40 \%$ had more than 4 assessments and about $15 \%$ had more than 8 assessments. Up to $60 \%$ of patients assessed PASI, DLQI and EQ-5D the same day as the biological agent was initiated. $30 \%$ had the assessment before switch to a biological agent 2 months before switch and the remaining, about $10 \%$, had the assessment more than 2 months before switch. About $1 / 3$ of patients had the follow-up assessment less than 2 months after switch, $1 / 3$ had it between 2 and 3 months and $1 / 3$ had the follow-up assessment more than 3 months after switch. (Follow-up times differed somewhat between measures where PASI had, on average, shorter and EQ-5D longer follow-up times.)

About $14 \%$ of patients had discontinued treatment with biological agents on follow-up, out of whom the majority, about $2 / 3$ of patients, discontinued treatment more than 2 months before the follow-up assessment.

There were statistically significant differences in PASI, DLQI and EQ-5D ( $\mathrm{p}<0.001)$ before switch to a biological agent and at follow-up (fig. 2, table 2). The majority of patients, $76 \%$, had PASI $>10$ and/or DLQI $>10$ before switch to a biological agent. One patient had a pretreatment PASI of 67.8, which is explained by the presence of erythroderma. Excluding the patient from analyses only changed results marginally.

The difference in EQ-5D increased with higher PASI and/or DLQI values before switch (table 3). 

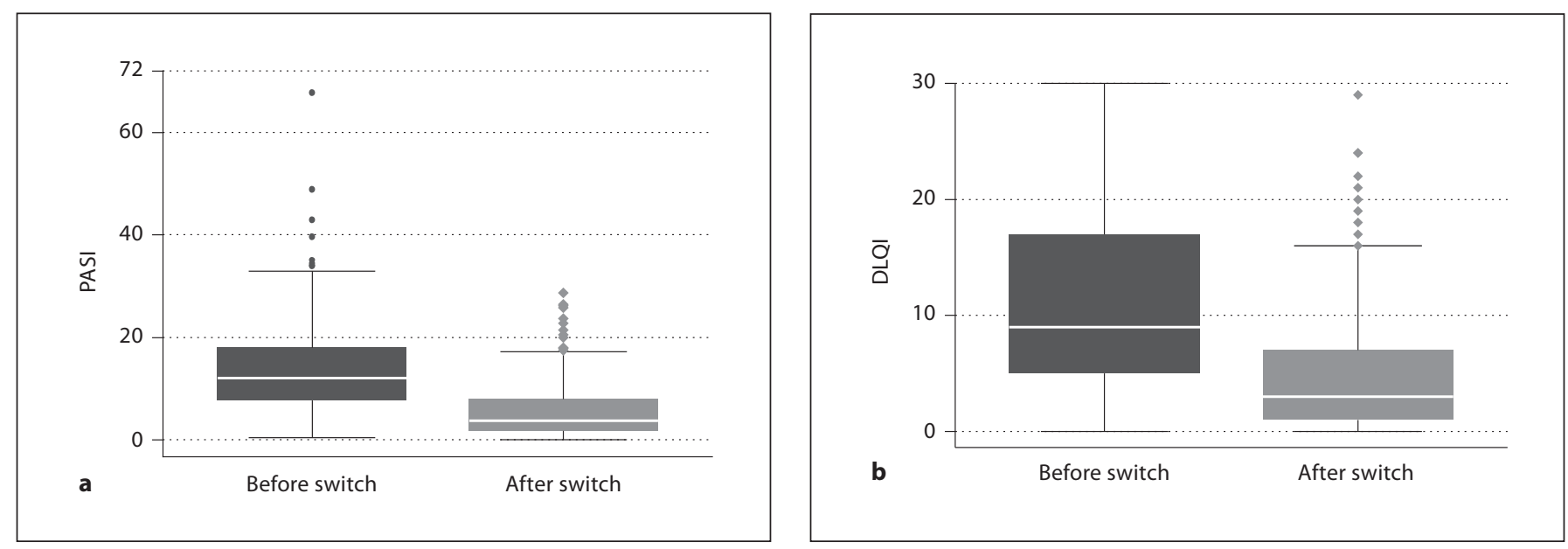

Fig. 2. Box plots of outcomes before and after switch to a biological agent. Box plots illustrate the distribution of PASI $(a, n=261)$, DLQI ( $\mathbf{b}, \mathrm{n}=251)$ and EQ-5D (c, $\mathrm{n}=229)$ before and after switch to a biological agent. The 'box' itself represents the lower quartile, median and upper quartile of data. The 'whiskers' represent the furthest observation which is within 1.5 interquartile range from the sides of the box. Observations not covered by the whiskers are outliers, marked with a dot in the graph.

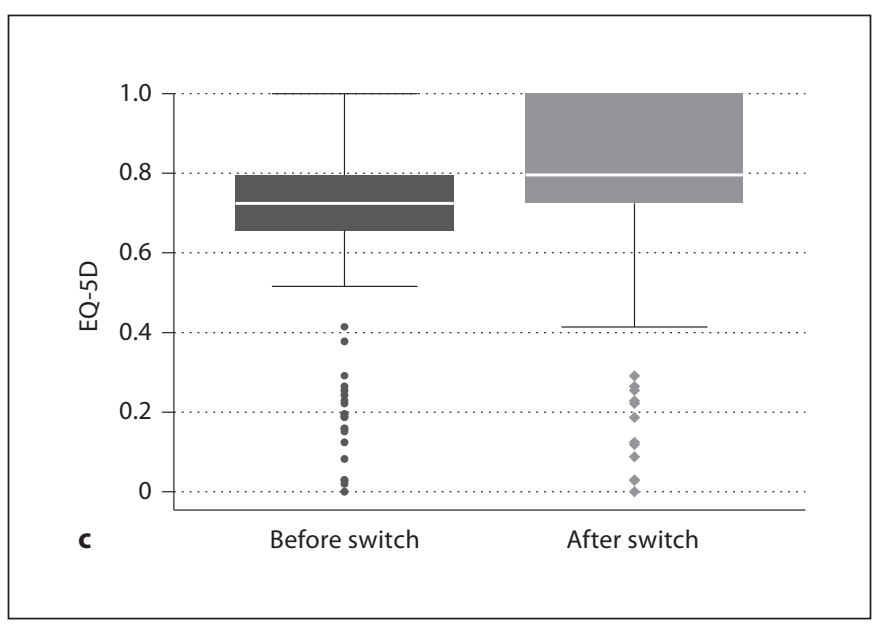

Table 2. Main results: outcomes before and after switch to a biological agent

\begin{tabular}{|c|c|c|c|c|c|c|c|}
\hline & median & mean $\pm \mathrm{SD}$ & median & mean $\pm \mathrm{SD}$ & median & mean $\pm \mathrm{SD}$ & $\mathrm{n}$ \\
\hline DLQI & 9 & $10.9 \pm 7.7$ & 3 & $5 \pm 5.8$ & 5 & $5.9 \pm 6.8$ & 251 \\
\hline EQ-5D & 0.725 & $0.683 \pm 0.259$ & 0.796 & $0.798 \pm 0.218$ & 0.069 & $0.115 \pm 0.239$ & 229 \\
\hline
\end{tabular}

Differences before and after in all outcome variables were significant at $\mathrm{p}<0.001$.

$62 \%$ of patients had a PASI 50 and $38 \%$ had a PASI 75. $20 \%$ had a PASI 90 . Patients who did not experience a reduction in PASI had limited change in EQ-5D.

There were no statistically significant differences between patients with or without nail involvement, in the change of EQ-5D ( $\mathrm{p}=0.922)$, or in EQ-5D, before ( $\mathrm{p}=$ $0.899)$ or after switch $(p=0.799)$. For uncommon clinical characteristics, the general tendency was that patients did not respond as well to biological agents, but results were not statistically significant which is likely to be due to 
Table 3. Subgroup analysis of change in EQ-5D before switch and at follow-up, subgroups based on PASI and DLQI intervals, PASI change intervals, presence of psoriatic arthropathy and PASI $>10$ and/or DLQI $>10$

\begin{tabular}{|c|c|c|c|c|c|c|c|c|}
\hline Subgroups & Intervals & \multicolumn{2}{|c|}{ EQ-5D before switch } & \multicolumn{2}{|c|}{ EQ-5D after switch } & \multicolumn{2}{|c|}{ EQ-5D change } & $\mathrm{n}$ \\
\hline \multirow{2}{*}{$\begin{array}{l}\text { PASI } \\
\text { (before switch) }\end{array}$} & PASI $>10$ & 0.725 & $0.658 \pm 0.271$ & 0.796 & $0.791 \pm 0.236$ & 0.071 & $0.132 \pm 0.247$ & 141 \\
\hline & Total & 0.725 & $\begin{array}{l}0.689 \pm 0.255 \\
*\end{array}$ & 0.796 & $\begin{array}{l}0.800 \pm 0.217 \\
\text { n.s. }\end{array}$ & 0.069 & $\begin{array}{l}0.111 \pm 0.236 \\
* *\end{array}$ & 223 \\
\hline \multirow{2}{*}{$\begin{array}{l}\text { DLQI } \\
\text { (before switch) }\end{array}$} & DLQI $>10$ & 0.656 & $0.549 \pm 0.271$ & 0.796 & $0.759 \pm 0.236$ & 0.143 & $0.210 \pm 0.246$ & 90 \\
\hline & Total & 0.725 & $\begin{array}{l}0.682 \pm 0.260 \\
* * *\end{array}$ & 0.796 & $\begin{array}{l}0.797 \pm 0.218 \\
* *\end{array}$ & 0.070 & $\begin{array}{l}0.115 \pm 0.239 \\
* * *\end{array}$ & 228 \\
\hline \multirow{2}{*}{$\begin{array}{l}\text { PASI } \\
\text { percentage } \\
\text { change }\end{array}$} & Not PASI 75 & 0.725 & $0.660 \pm 0.263$ & 0.796 & $0.747 \pm 0.231$ & 0.058 & $0.088 \pm 0.239$ & 139 \\
\hline & Reached PASI 75 & 0.796 & $0.737 \pm 0.236$ & 1 & $0.887 \pm 0.161$ & 0.107 & $0.149 \pm 0.228$ & 84 \\
\hline \multicolumn{2}{|c|}{$\begin{array}{l}\text { PASI } \leq 10 \text { and DLQI } \leq 10 \\
\text { PASI }>10 \text { and } / \text { or DLQI }>10 \\
\text { Total }\end{array}$} & 0.725 & $\begin{array}{l}0.796 \\
* * *\end{array}$ & 0.796 & $\begin{array}{l}0.796 \pm 0.221 \\
\text { n.s. }\end{array}$ & 0.07 & $\begin{array}{l}0.114 \pm 0.240 \\
* * *\end{array}$ & 220 \\
\hline
\end{tabular}

Absolute values. ${ }^{*} \mathrm{p}<0.1,{ }^{* *} \mathrm{p}<0.05,{ }^{* *} \mathrm{p}<0.01 ;$ n.s. $=$ not statistically significant.

limited sample size. The small sample of patients with erythroderma $(\mathrm{n}=10)$, however, showed a tendency $(\mathrm{p}=$ 0.081 ) of a better EQ-5D change (-0.23).

There were no significant differences in the change of outcomes between different biological agents, which may be due to limited sample size.

The regression analysis with EQ-5D change as outcome variable showed that $\operatorname{sex}(\mathrm{p}=0.775)$, age $(\mathrm{p}=0.115)$ and psoriatic arthropathy $(\mathrm{p}=0.102)$ were not statistically significant, whereas PASI $(\mathrm{p}=0.017)$ and DLQI $(\mathrm{p}<$ 0.001 ) were statistically significant. Sensitivity analysis showed similar results (data not shown).

\section{Discussion}

The clinical severity of skin and HRQOL improved after switch from conventional systemic treatment to a biological agent in a real-world setting. Patients with PASI $>10$ and/or DLQI $>10$ before switch experienced the greatest benefit in HRQOL.

The Swedish national registry PsoReg allowed for an analysis of the effectiveness of biological agents in clinical practice. The strength of this study is the real-world data, which made it possible to analyse benefits in different subgroups of patients. Published RCTs have assessed the efficacy of biological agents in selected trial populations in psoriasis. Our evaluation of real-world effectiveness adds new results in patient groups where characteristics may be different.

Since the study was not protocol driven and there were no mandatory follow-up visits in PsoReg, patients had different follow-up times. Some patients had even discontinued biological treatment on follow-up. This is a weakness which comes with an observational design as these factors, naturally, influenced outcomes; some patients may not have reached the full treatment effect, and some may have had a relapse of disease after discontinuing treatment. Furthermore, as psoriasis is a chronic disease with varying episodes of more or less severity, assessment of outcomes at one point in time may be misleading. Nonetheless, sensitivity analyses with a longer follow-up and excluding patients who had discontinued treatment showed that the main results were robust. However, a positive effect of a longer follow-up time in the sensitivity analyses may be offset due to an increasing number of patients discontinuing treatment. 
A recent review study concluded that there is a lack of large-scale real-world studies of psoriasis patients, particularly involving patient utility [8]. Observational studies including utility are mainly limited to cross-sectional analyses [27]. However, a number of RCTs have included EQ-5D. The EQ-5D change was between 0.12 and 0.21 and the DLQI change was 7.7-11.5 with follow-ups between 12 and 54 weeks $[4,28,29]$, compared to changes of 0.12 and 5.9 for EQ-5D and DLQI, respectively, in the present study. The EQ-5D change was in the lower end of previous RCTs, and the DLQI was slightly lower than RCT values. However, DLQI and EQ-5D changes of 2.35.7 and $0.09-0.22$, respectively, have shown to be the minimum important difference [15]. Our results were within these ranges. In a clinical trial of $50 \mathrm{mg}$ etanercept once weekly, 37.5\% of patients achieved PASI 75 after 12 weeks [28], which was similar to our study. In the clinical trial $71 \%$ had PASI 75 at week 24 [28]. Whereas the treatment goal in RCTs is often PASI 75, the treatment goal in clinical practice may be individual for each patient. There is no measure of patient treatment satisfaction available in PsoReg.

There are several reasons why we cannot expect that results in register-based studies match those in RCTs. One previously mentioned reason is different follow-up times; another is differences in patient characteristics. RCTs normally exclude comorbidities, and most trials demand a pretreatment PASI $>10$. Furthermore, some RCTs exclude patients because of adverse events, unsatisfactory response or because of protocol violation. Our study included patients with comorbidities and with other clinical characteristics of psoriasis than plaque psoriasis. 37\% of patients did not have a pretreatment value of PASI $>10$. Mean PASI scores at baseline in the studies mentioned above were between 14.5 and 21.4. It should also be acknowledged that the PASI can be affected by observer variation. In RCTs the assessment is made by a few trained physicians, whereas the PASI assessed in a national register of everyday clinical practice is dependent on several assessing physicians.

Despite the limitations of real-world data, results provide important knowledge about how treatment works in real-world practice. About a fourth of patients in this study who switched to a biological agent had pretreatment PASI $\leq 10$ and DLQI $\leq 10$. These patients did not benefit, on average, as much concerning HRQOL from biological agents as patients with severer psoriasis (PASI $>10$ and/or DLQI $>10$ ). This suggests that society could gain health (QALYs) by re-allocating resources from currently treated patients with low gains (low pretreatment
PASI and DLQI scores) to untreated patients with potentially high gains (patients with high PASI and DLQI scores). Let us assume that about $25 \%$ of the approximately 1,500 patients currently treated with biological agents for psoriasis in Sweden would have PASI $\leq 10$ and DLQI $\leq 10$ with a utility (EQ-5D gain) of approximately 0.02 . By re-allocating resources and treating patients with PASI $>10$ and/or DLQI $>10$ and potential utility of 0.14 , we would gain 45 QALYs $(0.25 \cdot 1,500 \cdot 0.12=45)$. This naïve estimate assumes that patients with PASI $>10$ and/or DLQI $>10$ currently treated with conventional treatments would respond as patients in our study and that utility would be constant over a year. Even though individual patients with low PASI and DLQI values may suffer from their disease, this study shows that, on average, patients with PASI $>10$ and/or DLQI $>10$ tend to have the highest benefits, suggesting that this patient group would be most cost-effective to treat.

When new treatments are introduced in clinical practice, it may be challenging to target the unselected patient groups for which these treatments are effective. Future research should investigate potential over- and undertreatment of biological agents in patients with moderate to severe psoriasis in clinical practice.

\section{Acknowledgements}

The authors wish to thank all those who helped to create PsoReg, all patients and all health care personnel and the PsoReg steering board. We wish to thank our reviewers for the beneficial comments on the manuscript.

\section{Disclosure Statement}

PsoReg has received financial support from Swedish Board of Health and Welfare, Swedish Association of Local Authorities and Regions, Västerbotten County Council, Abbott, Jansen-Cilag, Leo Pharma, MSD and Pfizer. This research has, in addition, received financial support from Abbott, Jansen-Cilag, Leo Pharma and Pfizer. The sponsors had no access to data. Data collection, study design, interpretation, and analysis have been carried out with authors' independence. None of the authors has a conflict of interest to declare.

References

1 Schmitt J, Zhang Z, Wozel G, Meurer M, Kirch W: Efficacy and tolerability of biologic and nonbiologic systemic treatments for moderate-to-severe psoriasis: meta-analysis of randomized controlled trials. Br J Dermatol 2008; 159:513-526. 
-2 Bansback N, Sizto S, Sun H, Feldman S, Willian MK, Anis A: Efficacy of systemic treatments for moderate to severe plaque psoriasis: systematic review and meta-analysis. Dermatology 2009;219:209-218.

-3 Revicki D, Willian MK, Saurat JH, Papp KA, Ortonne JP, Sexton C, Camez A: Impact of adalimumab treatment on health-related quality of life and other patient-reported outcomes: results from a 16-week randomized controlled trial in patients with moderate to severe plaque psoriasis. Br J Dermatol 2008;158:549-557.

-4 Shikiar R, Heffernan M, Langley RG, Willian MK, Okun MM, Revicki DA: Adalimumab treatment is associated with improvement in health-related quality of life in psoriasis: patient-reported outcomes from a phase II randomized controlled trial. J Dermatolog Treat 2007;18:25-31.

5 Reich K, Griffiths CEM: The relationship between quality of life and skin clearance in moderate-to-severe psoriasis: lessons learnt from clinical trials with infliximab. Arch Dermatol Res 2008;300:537-544.

6 Grossman J, Mackenzie FJ: The randomized controlled trial: gold standard, or merely standard? Perspect Biol Med 2005;48:516534.

7 Rosen M, Axelsson S, Lindblom J: When can RCTs and observational intervention studies mislead us and what can we do about it? Int J Clin Pract 2009;63:1562-1564.

-8 Langham S, Langham J, Goertz HP, Ratcliffe M: Large-scale, prospective, observational studies in patients with psoriasis and psoriatic arthritis: a systematic and critical review. BMC Med Res Methodol 2011;11:32.

9 Barzi S, Befrits G, Bergman B, et al: Läkemedelsbehandling av psoriasis - ny rekommendation. Information from the Medical Products Agency, 2011.

10 Alenius GM, Bergbrant IM, Bergström K, et al: Behandling av psoriasis - behandlingsrekommendation. Information from the Medical Products Agency, 2006, pp 5-16.
Garduno J, Bhosle MJ, Balkrishnan R, Feldman SR: Measures used in specifying psoriasis lesion(s), global disease and quality of life: a systematic review. J Dermatolog Treat 2007;18:223-242.

12 Bronsard V, Paul C, Prey S, Puzenat E, Gourraud $\mathrm{PA}$, Aractingi $\mathrm{S}$, Aubin F, Bagot $\mathrm{M}$, Cribier B, Joly P, Jullien D, Le Maitre M, Richard-Lallemand MA, Ortonne JP: What are the best outcome measures for assessing quality of life in plaque type psoriasis? A systematic review of the literature. J Eur Acad Dermatol Venereol 2010;24(suppl 2):17-22.

3 Both H, Essink-Bot ML, Busschbach J, Nijsten T: Critical review of generic and dermatology-specific health-related quality of life instruments. J Invest Dermatol 2007;127: 2726-2739.

14 Lewis V, Finlay AY: 10 years experience of the dermatology life quality index (DLQI). J Investig Dermatol Symp Proc 2004;9:169-180.

15 Shikiar R, Willian MK, Okun MM, Thompson CS, Revicki DA: The validity and responsiveness of three quality of life measures in the assessment of psoriasis patients: results of a phase II study. Health Qual Life Outcomes 2006;4:71.

16 Basra MK, Fenech R, Gatt RM, Salek MS, Finlay AY: The dermatology life quality index 1994-2007: a comprehensive review of validation data and clinical results. Br J Dermatol 2008;159:997-1035.

17 Shikiar R, Bresnahan BW, Stone SP, Thompson C, Koo J, Revicki DA: Validity and reliability of patient reported outcomes used in psoriasis: results from two randomized clinical trials. Health Qual Life Outcomes 2003; 1:53.

18 Norlin JM, Steen Carlsson K, Persson U, Schmitt-Egenolf M: Analysis of three outcome measures in moderate to severe psoriasis - a registry based study of 2,450 patients. Br J Dermatol 2012;166:797-802.

19 Drummond MF, Sculpher M, Torrance G, O'Brian B, Stoddart G: Methods for the Economic Evaluation of Health Care Programmes. New York, Oxford University Press, 2005.

20 Schmitt-Egenolf M: PsoReg - the Swedish Registry for Systemic Psoriasis Treatment. The registry's design and objectives. Dermatology 2007;214:112-117.
1 EuroQol - a new facility for the measurement of health-related quality of life. The EuroQol Group. Health Policy 1990;16:199-208.

22 Dolan P: Modeling valuations for EuroQol health states. Med Care 1997;35:1095-1108.

23 Finlay AY, Khan GK: Dermatology life quality index (DLQI) - a simple practical measure for routine clinical use. Clin Exp Dermatol 1994;19:210-216.

24 Fredriksson T, Pettersson U: Severe psoriasis - oral therapy with a new retinoid. Dermatologica 1978;157:238-244.

25 Mrowietz U, Kragballe K, Reich K, Spuls P, Griffiths CE, Nast A, Franke J, Antoniou C, Arenberger P, Balieva F, Bylaite M, Correia O, Dauden E, Gisondi P, Iversen L, Kemeny L, Lahfa M, Nijsten T, Rantanen T, Reich A, Rosenbach T, Segaert S, Smith C, Talme T, Volc-Platzer B, Yawalkar N: Definition of treatment goals for moderate to severe psoriasis: a European consensus. Arch Dermatol Res 2011;303:1-10.

-26 Rosen CF, Mussani F, Chandran V, Eder L, Thavaneswaran A, Gladman DD: Patients with psoriatic arthritis have worse quality of life than those with psoriasis alone. Rheumatology (Oxford) 2012;51:571-576.

27 Augustin M, Krüger K, Radtke MA, Schwippl I, Reich K: Disease severity, quality of life and health care in plaque-type psoriasis: a multicenter cross-sectional study in Germany. Dermatology 2008;216:366-372.

28 Reich K, Segaert S, van de Kerkhof P, Durian C, Boussuge MP, Paolozzi L, Wajdula J, Boggs R: Once-weekly administration of etanercept $50 \mathrm{mg}$ improves patient-reported outcomes in patients with moderate-to-severe plaque psoriasis. Dermatology 2009; 219:239-249.

29 Dauden E, Griffiths CE, Ortonne JP, Kragballe K, Molta CT, Robertson D, Pedersen R, Estojak J, Boggs R: Improvements in patientreported outcomes in moderate-to-severe psoriasis patients receiving continuous or paused etanercept treatment over 54 weeks: the Crystel study. J Eur Acad Dermatol Venereol 2009;23:1374-1382. 